\title{
Análisis comparativo de las modificaciones hemodinámicas y del flujo sanguíneo renal (FSR) durante la nefrectomía abierta y laparoscópica. Modelo experimental
}

\author{
Linares Quevedo $\mathrm{AI}^{1}$, Burgos Revilla $\mathrm{FJ}^{2}$, Villafruela Sanz $\mathrm{JJ}^{4}$ Zamora Romero $\mathrm{J}^{3}$, \\ Pascual Santos $\mathrm{J}^{3}$, Marcén Letosa $\mathrm{R}^{4}$, Cuevas Sánchez $\mathrm{B}^{4}$, Correa Gorospe $\mathrm{C}^{4}$. \\ ${ }^{1}$ Servicio de Urología. Hospital de Fuenlabrada. ${ }^{2}$ Servicios de Urología, ${ }^{3}$ Nefrología y ${ }^{4}$ Unidad de Apoyo a la \\ Investigación. Hospital Ramón y Cajal. Universidad de Alcalá. Madrid.
}

Actas Urol Esp. 2007;31(4):382-393

\section{RESUMEN}

ANÁLISIS COMPARATIVO DE LAS MODIFICACIONES HEMODINÁMICAS Y DEL FLUJO SANGUÍNEO RENAL (FSR) DURANTE LA NEFRECTOMÍA ABIERTA Y LAPAROSCÓPICA. MODELO EXPERIMENTAL

Introducción: $\mathrm{El}$ aumento de la presión intraabdominal (PIA) por encima de $10 \mathrm{mmHg}$ conlleva una reducción del flujo sanguíneo renal (FSR). El neumoperitoneo inducido durante las técnicas laparoscópicas, condiciona presiones intraabdominales (PIA) próximas a $15 \mathrm{mmHg}$, circunstancia que reduce el FSR, la diuresis y el filtrado glomerular $(\mathrm{FG})$.

Objetivo: Analizar comparativamente en un modelo experimental en cerdo, las modificaciones del FSR, la diuresis y el FG inducidas por las nefrectomías abierta y laparoscópica.

Material y métodos: Un total de 30 cerdos (peso medio $=22,6+3,2 \mathrm{Kg}$ ) han sido sometidos a nefrectomía izquierda: 15 por laparoscopia y 15 por vía abierta, como donantes vivos en un modelo de autotrasplante renal. Se midió la diuresis y el FG basales y a los 30 y 60 min del inicio de la nefrectomía. El FSR medio se determinó mediante sonda electromagnética en la arteria renal principal durante la primera hora de la cirugía.

Resultados: Se objetivó una reducción significativa del FSR $(80+2,7$ vs $262+3 \mathrm{ml} / \mathrm{min})(\mathrm{p}<0,005)$ durante la nefrectomía laparoscópica frente a la abierta. También se demostró una disminución significativa de la diuresis (42\%) y del FG (38\%) del grupo laparoscópico frente al abierto.

Conclusiones: La nefrectomía laparoscópica conlleva una disminución significativa del FSR, circunstancia potencialmente trascendente en el TR con donante vivo, así como del FG y de la diuresis.

Palabras clave: Flujo sanguíneo renal. Nefrectomía. Laparoscopia.

\section{ABSTRACT}

\section{COMPARATIVE ANALYSIS ABOUT HEMODYNAMIC AND RENAL BLOOD FLOW EFFECTS DURING OPEN} VERSUS LAPAROSCOPIC NEPHRECTOMY. AN EXPERIMENTAL STUDY

Background: The increase of intraabdominal pressure to $10 \mathrm{mmHg}$ provokes a decrease of renal blood flow (RBF). Pneumoperitoneum during laparoscopic techniques with intra-abdominal pressure (IAP) to $15 \mathrm{mmHg}$, results in a decrease in RBF, urine output and glomerular filtration rate (GFR).

PURPOSE: Analyze the changes in RBF, urine output an GFR in a porcine experimental model during open vs laparoscopic nephrectomy.

Materials and methods: 30 pigs (medium weigh $=22.6+3.2 \mathrm{Kg}$ ) were divided into two groups: Laparoscopic nephrectomy was performed using 15 pigs and open nephrectomy in 15 pigs, following a living donor nephrectomy autotransplantation model. Study parameters were urine volume and GFR baseline values, 30 and 60 minutes during nephrectomy. RBF was measured using an electromagnetic flow catheter around the main renal artery during the initial 60 minutes of nephrectomy.

Results: The laparoscopic technique was associated with a significant reduction of RBF $(80+2.7$ vs $262+3$ $\mathrm{ml} / \mathrm{min})(\mathrm{p}<0.005)$, diuresis $(42 \%)$ and GFR $(38 \%)$, vs the open group.

Conclusions: Laparoscopic nephrectomy involves a significant reduction of RBF, GFR and diuresis, which is potentially transcendent in living donor nephrectomy and kidney transplantation. 
$\mathrm{E}$ n sus primeros tiempos, la laparoscopia se limitaba a procedimientos diagnósticos de corta duración. Durante estos breves procesos, no se observaban las manifestaciones fisiológicas relacionadas con el neumoperitoneo.

A medida que las intervenciones laparoscópicas se hacían más complejas y prolongadas, se evidenciaron las consecuencias fisiopatológicas de la insuflación del abdomen con $\mathrm{CO}_{2}$. Un neumoperitoneo de larga duración puede afectar a varios sistemas homeostáticos ${ }^{1,2}$.

A medida que la insuflación de gas aumenta la presión intraabdominal (PIA), la resistencia vascular sistémica se eleva. Esto parece ser un fenómeno directo de compresión que afecta, principalmente a la circulación esplácnica ${ }^{3}$. Las modificaciones de la resistencia tienen lugar tanto en los capilares como en los vasos de capacitancia de los sistemas venoso y arterial ${ }^{4-8}$. El flujo sanguíneo a los órganos intraabdominales, con excepción de la glándula suprarrenal, disminuye hasta los $20 \mathrm{mmHg}$ de PIA en modelos animales ${ }^{9}$.

La magnitud del efecto de la PIA en la resistencia vascular sistémica depende del estado volumétrico del sujeto ${ }^{9}$.

$\mathrm{El}$ neumoperitoneo tiene efecto sobre distintos órganos:

\section{EFECTOS CARDIOVASCULARES}

El gasto cardiaco (GC) está limitado por el retorno venoso: el corazón no puede bombear más sangre de la que recibe. A niveles bajos de PIA (menos de $10 \mathrm{mmHg}$ ), en realidad, el retorno venoso aumenta debido a la "autotransfusión" desde unos vasos abdominales parcialmente vacíos, y por tanto, el GC aumenta también. Pero a medida que la PIA se eleva por encima de los 20 $\mathrm{mmHg}$, el retorno venoso y el GC tienden a disminuir ${ }^{3}$.

La tensión arterial media (TAM) es producto del GC y de la resistencia arterial. El equilibrio entre ambos, a una PIA $<20 \mathrm{mmHg}$, eleva la TAM. Pero cuando la PIA se sitúa por encima de los 40 mmHg, la tensión arterial (TA) cae, ya que el GC desciende más de lo que aumenta la resistencia arterial ${ }^{10}$. Por otra parte, la hipertensión en el curso de una laparoscopia puede deberse a hipoxemia, a hipercapnia, o a PIA moderadamente elevada ${ }^{11,12}$.
La absorción de $\mathrm{CO}_{2}$, el gas que se emplea con más frecuencia, hace sentir sus efectos en varios puntos del organismo, a menudo con resultados opuestos. Los efectos directos del $\mathrm{CO}_{2}$ son principalmente inhibitorios sobre el sistema cardiovascular: disminución de la frecuencia cardiaca, de la contractilidad cardiaca y de la resistencia vascular $^{7}$. Sin embargo, la estimulación del sistema nervioso simpático por parte del $\mathrm{CO}_{2}$ contrarresta estos efectos. Los eferentes simpáticos y las catecolaminas circulantes aumentan la frecuencia y la contractilidad cardiacas y la resistencia vascular. Si se permite que se desarrolle acidosis, se pueden potenciar los efectos parasimpáticos. Fundamentalmente, una moderada hipercapnia produce un aumento del GC y de la TA, y un descenso de la resistencia vascular sistémica ${ }^{6,7}$.

\section{EFECTOS SOBRE EL RIÑÓN}

El neumoperitoneo causa disfunción renal reversible, que se hace más pronunciada, cuanto más largo sea el período de insuflación ${ }^{13,14}$. Algunos estudios también han descrito fracaso renal oligúrico en pacientes con elevación de la PIA secundaria a hemorragia abdominal traumática, ascitis hepática o procesos malignos intraabdominales ${ }^{8,9}$.

Así, aunque se ha descrito el deterioro de la función renal en relación con diferentes procesos que cursan con elevaciones de la PIA, la causa no está claramente establecida.

Se han propuesto varios mecanismos responsables del daño renal: la compresión del parénquima renal, del flujo arterial y venoso renal, de la presión venosa central (PVC), la disminución del GC y la obstrucción ureteral:

- La compresión del parénquima renal ha sido descrita como causante de deterioro de la función renal e hipertensión ${ }^{13,}{ }^{14}$. Page ${ }^{15}$, fue el primero en demostrar que la compresión del parénquima renal, se relacionaba con la aparición de hipertensión renal, y de una reducción de la diuresis. En su descripción original, la aparición de una "perinefritis constrictiva" ocasionaba isquemia renal e hipertensión secundaria, que se atribuyó a la presencia de una sustancia presora que denominó "activador de renina".

Más adelante, Skinner ${ }^{16}$ afirmó que este efecto estaba mediado por la renina, al demostrar 
una elevación de la actividad de la renina plasmática, después de una compresión del riñón por balón a tensiones de 15 a $40 \mathrm{mmHg}$. Este incremento de la actividad de renina plasmática con el aumento de la PIA ha sido ampliamente referido en la literatura ${ }^{17}$.

La compresión renal directa por presión intrabdominal de $>10 \mathrm{mmHg}$, causa una disminución del FG y del flujo sanguíneo renal (FSR) ${ }^{18}$, con la consiguiente oliguria ${ }^{19}$.

Razvi et al. ${ }^{17}$, demostraron, que la compresión del parénquima renal, causaba un efecto directo y reversible sobre el FG, al igual que la compresión arterial y venosa renal. La compresión renal ejercida por el neumoperitoneo, no sólo afectaría al FG, sino también ocasionaría la compresión de las nefronas, y ambos mecanismos contribuirian a la disminución de la diuresis. No se encontraron alteraciones histopatológicas ${ }^{2}$. Estos mismos autores demostraron que una compresión renal a $15 \mathrm{mmHg}$, condiciona una disminución de la diuresis del 63\%, del FG en un 21\%, y del FSR en un $26 \%$.

Así, la compresión del parénquima por sí sola, puede ser responsable de la disminución reversible del FG, del FSR y de la liberación de renina durante una insuflación laparoscópica.

- La compresión de las venas renales. Durante un neumoperitoneo, la PIA llega al doble de la presión de la vena cava inferior ${ }^{6}$. Las alteraciones en la presión de las venas renales, son prácticamente paralelas a las alteraciones de la PIA ${ }^{20}$. La disminución de la diuresis, a una PIA superior a $15 \mathrm{mmHg}$, fue paralela a una reducción del flujo venoso renal. La perfusión con dopamina no logró aumentar la diuresis ${ }^{21}$.-

- Flujo sanguíneo arterial renal. El FSR, es estable a una PIA por debajo de $10 \mathrm{mmHg}$. Sin embargo, en modelos experimentales en cerdo, a presiones de $20 \mathrm{mmHg}$ hay casi un 15\% de disminución del flujo arterial renal, de un 58 a un $60 \%$ de caída de la perfusión cortical, un $64 \%$ de reducción del FG y un 50\% de disminución de la diuresis ${ }^{6,22,23}$

Cuando se realizó una compresión unilateral, la diuresis disminuyó en un $25 \%$. Después de la desinsuflación, la perfusión cortical renal vuelve a sus niveles normales, aunque la diuresis continúa disminuida una hora más. Así, la compre- sión renal no es el único factor causante de la oliguria, sino que actúa conjuntamente con los mecanismos mencionados.

- Compresión venosa central. Kirsch et al. ${ }^{24}$, tras los estudios realizados en un modelo experimental en ratas, sugieren que probablemente la oliguria inducida por el neumoperitoneo, esté relacionada con una disminución del flujo vascular renal causada por compresión venosa central. Demostraron, que con una insuflación a 10 mmHg, el flujo de la cava inferior se reducía en un $93 \%$ y el flujo aórtico en un $45 \%$. Las presiones elevadas de la cava inferior, dan como resultado una disminución del FSR y del FG, todo lo cual, lleva a una reducción de la diuresis.

- Disminución del GC. El FSR representa aproximadamente el $25 \%$ del gasto cardíaco. El flujo sanguíneo cortical (4-5 $\mathrm{ml} / \mathrm{Kg} / \mathrm{min})$ y de la médula renal $(0,2-0,03 \mathrm{ml} / \mathrm{Kg} / \mathrm{min})$ es elevado en comparación con otros órganos. Este flujo es necesario para mantener el FG y la reabsorción y secreción tubular ${ }^{8,9}$.

Los pacientes con cardiopatías sometidos a una colecistectomía laparoscópica exhibieron una producción de orina notablemente reducida en comparación con los pacientes sanos ${ }^{25}$. Vukasin et al. ${ }^{20}$, utilizaron el modelo de perro para demostrar una disminución concomitante en la producción de orina y el GC durante la insuflación con $\mathrm{CO}_{2}$ a intervalos de tiempo variables.

- Obstrucción ureteral. Las urografias realizadas durante la insuflación demostraron la ausencia de obstrucción. La colocación de catéteres ureterales no modificaban las alteraciones hemodinámicas que sufre el riñón durante el aumento de PIA. Ello parece indicar que la compresión ureteral durante la laparoscopia tiene un escaso papel en la alteración de la función renal inducida por el neumoperitoneo, y no evita la oliguria que tiene lugar durante el neumoperitoneo.

Estos mecanismos que generan alteración de la función renal son potencialmente trascendentes en distintas situaciones clínicas: entre otros, pacientes con insuficiencia renal, y en el riñón de donante vivo.

El trasplante renal (TR) de donante vivo representa una alternativa para hacer frente a la escasez de órganos para el TR. Distintos mecanismos han sido implicados en la disfunción inicial de los 
injertos extraídos por vía laparoscópica, entre ellos el mayor tiempo de isquemia caliente y los efectos que el neumoperitoneo ejerce sobre el riñón ${ }^{21,26}$.

El interés práctico de este estudio consiste en conocer las modificaciones hemodinámicas y del FSR sufridas por el riñón debido al neumoperitoneo durante el proceso de nefrectomía laparoscópica, y sus consecuencias sobre el FG y diuresis que condicionarán la función renal tras el trasplante.

Para llevar a cabo el estudio se eligió un modelo experimental y no clínico. De esta manera se tiene un control adecuado de todas las variables que intervienen. Además, se escogió un animal de experimentación grande, como el cerdo, que guarda una gran similitud con el humano.

\section{MÉTODOS}

Los animales de estudio son 30 cerdos de raza ibérica, cuyo peso medio fue de $22.6+3,2 \mathrm{Kg}$ (Rango 19-31 Kg).

Se estudiaron dos grupos de 15 cerdos cada uno:

- GRUPO ABIERTO ( $n=15$ cerdos). Utilizado como "GRUPO CONTROL" en el que el abordaje renal se realizó por cirugía abierta convencional.

- GRUPO LAP ( $n=15$ cerdos). Utilizado como “GRUPO ESTUDIO” en el que el abordaje se practicó por vía laparoscópica.

El estudio experimental realizado se basa en el siguiente diseño:

\section{Premedicación y anestesia general del} animal. Son sometidos a un período de ayuno de 12 horas previo a la cirugía, pero con ingesta libre de agua. Se realiza en todos ellos una premedicación con Ketamina intramuscular (10 $\mathrm{mg} / \mathrm{Kg}$ ) e inducción anestésica con Tiopental sódico intravenoso (10 mg/Kg) y Atropina $(0,01$ $\mathrm{mg} / \mathrm{Kg}$ ). Se procede a la intubación endotraqueal y ventilación controlada del animal. La anestesia se mantiene con Halotano al 0,9-1,25\% y $\mathrm{N}_{2} \mathrm{O}$ mezclado con $\mathrm{O}_{2}$. La analgesia intraoperatoria se

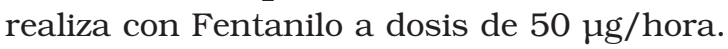

Se administra de forma preoperatoria Cefazolina 1 gramo intravenoso en dosis única. La hidratación del animal durante la cirugía se realiza mediante la administración de suero glucosalino a dosis de $10 \mathrm{ml} / \mathrm{Kg} /$ hora.
2. Abordaje Quirúrgico. En el Grupo Abierto: incisión de lumbotomía izquierda bajo la décima segunda costilla en los 15 animales. En el grupo LAP se realiza abordaje laparoscópico transperitoneal e insuflación con $\mathrm{CO}_{2}$ para mantener una PIA constante de $15 \mathrm{mmHg}$.

3. Monitorización en ambos grupos de la TA y PVC. La monitorización hemodinámica de los animales, se inicia mediante la disección de la región femoral derecha, y colocación de un catéter arterial en la arteria femoral derecha, y el mismo tipo de catéter en la vena femoral derecha. Así, se monitorizan de forma continua la TA y la PVC. La monitorización electrocardiográfica también se lleva a cabo de forma continua mediante las derivaciones II y IV.

4. Determinaciones analiticas. Las muestras de sangre (Creatinina plasmática) durante la intervención se toman a través del catéter central colocado en la vena femoral derecha en los dos grupos: basal (previo al comienzo de la nefrectomía izquierda) y a los a los 60 minutos de inicio de la cirugía tanto abierta como laparoscópica.

Las determinaciones de orina (creatinina) y diuresis se realizan mediante sondaje vesical 30 minutos antes del comienzo de cirugía, y a los 30 y 60 minutos durante ambas cirugías.

5. Medidas del FSR. Las medidas de FSR, se realizan mediante la colocación del transductor de la sonda electromagnética alrededor de la arteria renal. Las modificaciones en la perfusión renal durante el neumoperitoneo mediante las determinaciones del índice de resistencia (IR) y las velocidades de perfusión sistólica y diastólica mediante eco doppler, que se obtienen en los siguientes momentos definidos:

- En el grupo ABIERTO (CONTROL). Colocación de sonda electromagnética durante la nefrectomía izquierda abierta una vez que el FSR se estabiliza durante la nefrectomía. Sirven como determinación basal del animal. Nueva determinación a los 60 minutos de la cirugía abierta.

- En el grupo LAP (ESTUDIO). Realización de eco doppler previo al inicio de la intervención y durante la cirugía laparoscópica tras la insuflación a los 5 minutos. 
La determinación con sonda electromagnética del FSR se realiza tanto basal, como a los $60 \mathrm{mi}-$ nutos de insuflación. Además se realizan determinaciones seriadas en dos fases: una tras estabilización del neumoperitoneo, a los minutos 0 , 1,5, y 10 y otra tras desinsuflación a los minutos $0,1,5$ y 10. Los resultados obtenidos traducen las modificaciones del flujo arterial renal sufridas durante la laparoscopia.

\section{Análisis estadístico de los resultados.} Como estimador de la tendencia central de las variables se utilizó la media, y como estimador de la dispersión se empleó la desviación estándar. Para comparar la diferencia entre las medias, se utilizó el test de la t de Student.

Para todas las comparaciones se empleó un nivel de significación estadística del 95\%.

El estudio estadístico se realizó con el programa SAS 8.2 y la realización de los gráficos mediante el programa SPSS 6.2.

\section{RESULTADOS}

\section{Efectos hemodinámicos}

Los valores de TAM y PVC en el grupo LAP fueron significativamente superiores que los de TAM y PVC en el grupo ABIERTO. (Tabla 1)

Tabla 1

Valores de TAM y PVC media en cada grupo. Se analizan comparativamente las cifras de TAM y PVC media durante la primera hora de neumoperitoneo y en los siguientes momentos: basal, 5, 30 y 60 minutos.

\begin{tabular}{lccc}
\hline & $\begin{array}{c}\text { G. ABIERTO } \\
\mathbf{n = 1 5} \\
\text { media } \pm \text { d.s. }\end{array}$ & $\begin{array}{c}\text { G. LAP } \\
\mathbf{n = 1 5} \\
\text { media } \pm \text { d.s. }\end{array}$ & $\begin{array}{c}\text { Valor } \\
\text { de } \mathbf{~}\end{array}$ \\
\hline TAM & & & \\
mmHg & $\mathbf{8 3 , 1} \pm \mathbf{0 , 6}$ & $\mathbf{8 8 , 1} \pm \mathbf{1 , 1}$ & $<\mathbf{0 , 0 0 1 *}$ \\
Basal & $83,1 \pm 1,2$ & $84,0 \pm 3,6$ & 0,53 \\
$5 \mathrm{~m}$ & $82,8 \pm 1,3$ & $81,0 \pm 4,7$ & 0,32 \\
$30 \mathrm{~m}$ & $83,4 \pm 1,5$ & $86,0 \pm 5,5$ & 0,13 \\
$60 \mathrm{~m}$ & $83,2 \pm 1,2$ & $100,0 \pm 4,9$ & $<0,0001$ \\
& & & \\
PVC & & & $<0,001 *$ \\
cmH $\mathbf{2}$ & $\mathbf{1 2 , 4} \pm \mathbf{0 , 1}$ & $\mathbf{2 1 , 5} \pm \mathbf{0 , 7}$ & $<0,2$ \\
Basal & $12,4 \pm 0,4$ & $13,9 \pm 1,6$ & 0,0001 \\
$5 \mathrm{~m}$ & $12,2 \pm 0,5$ & $26,0 \pm 2,0$ & $<0,0001$ \\
$30 \mathrm{~m}$ & $12,4 \pm 0,4$ & $25,0 \pm 2,0$ & $<0,0001$ \\
$60 \mathrm{~m}$ & $12,7 \pm, 4$ & $21,0 \pm 1,8$ & $<0,0001$ \\
\hline
\end{tabular}

* Diferencia de TAM y PVC media significativa durante la primera hora de cirugía en el G. ABIERTO y G. LAP.
En el grupo LAP se produjo un ligero descenso de la TAM a los 5 minutos de la insuflación de $4 \mathrm{mmHg}$, para ir posteriormente aumentando de forma lentamente progresiva hasta elevarse 16 $\mathrm{mmHg}$ respecto al valor basal. Este incremento se hace significativo respecto a la TAM basal del grupo LAP transcurrida 1 hora desde la insuflación, cuando se registra un incremento del 18\% de la TAM (Fig. 1).

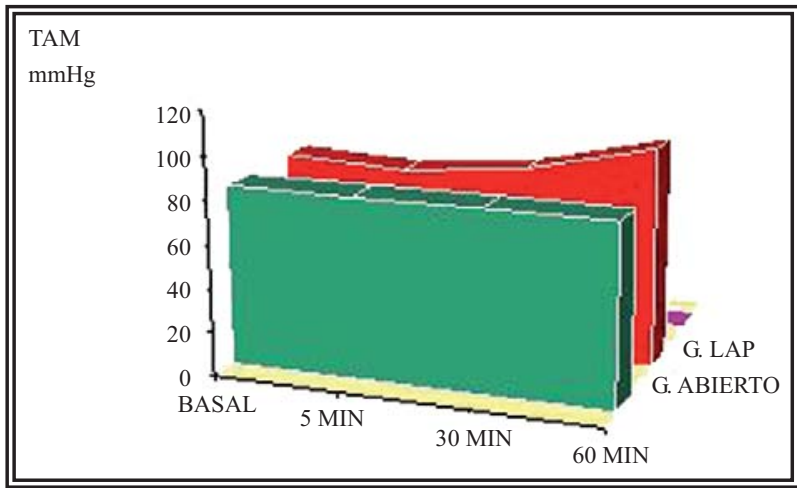

FIGURA 1. Evolución de la TAM durante la primera hora en el grupo de nefrectomía abierta y durante la primera hora de neumoperitoneo en el grupo de nefrectomía laparoscópica.

La PVC se incrementa durante el neumoperitoneo, pero a diferencia de lo que sucede con los valores de TAM, esa elevación ya es significativa tras 5 minutos de insuflación respecto al valor basal de PVC del grupo LAP y ABIERTO. Se registra un incremento del $100 \%$ a los 5 minutos, donde alcanza el máximo de $26 \mathrm{~cm} \mathrm{H}_{2} \mathrm{O}$, manteniéndose en niveles estables durante la siguiente media hora, para ir descendiendo progresivamente hasta los 60 minutos, pero siempre registrando valores significativamente superiores a los del grupo ABIERTO (Fig. 2).

\section{Efectos sobre el FSR}

El grupo LAP experimentó una disminución significativa del FSR respecto al FSR del grupo ABIERTO.

El valor de FSR, medido mediante sonda electromagnética sobre la arteria renal, durante la nefrectomía abierta fue de 262,0+3,1 $\mathrm{ml} / \mathrm{min}$, mientras que en el caso de la nefrectomía laparoscópica descendió alcanzándose una valor medio de $80,0+2,7 \mathrm{ml} / \mathrm{min}$, lo que supone una disminución del 69\% del FSR respecto al grupo ABIERTO (Fig. 3). 


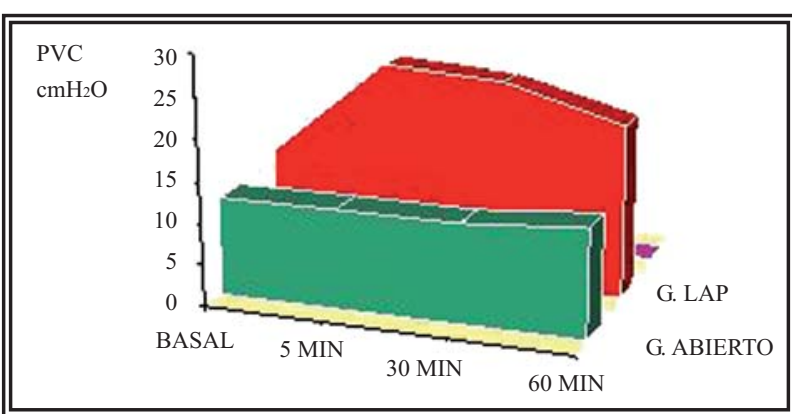

FIGURA 2. Evolución de la PVC durante la primera hora en el grupo de nefrectomía abierta y durante la primera hora de neumoperitoneo en el grupo de nefrectomia laparoscópica.

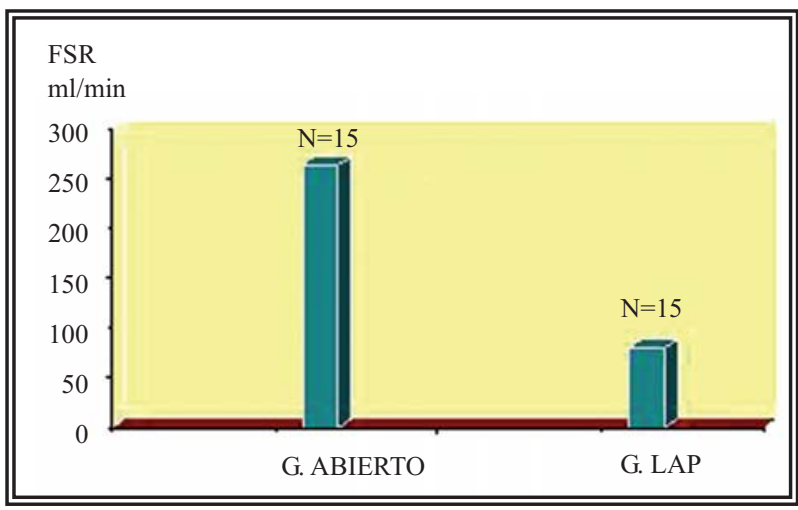

FIGURA 3. Modificaciones del FSR medio durante la primera hora de la nefrectomía abierta y laparoscópica.

Durante la fase de insuflación se objetivó un descenso inmediato y significativo del FSR que se hace máximo a los 5 minutos de la inducción del neumoperitoneo; posteriormente, se produce un pequeño ascenso que se mantiene hasta el final del proceso con un FSR en torno al 43\% del basal (Fig. 4).

Tras iniciar la desinsuflación, se produce un rápido ascenso del FSR, hasta alcanzar prácticamente los niveles basales a partir del primer minuto de haberse iniciado la desinsuflación. A partir de los 5 minutos se objetiva un fenómeno de "rebote": el FSR se incrementa hasta un 40\% respecto al FSR basal (Fig. 5).

$\mathrm{El}$ análisis de las modificaciones de la perfusión renal mediante eco-doppler, sufridas durante la nefrectomía laparoscópica reveló que existía una disminución significativa respecto a la basal, de la velocidad diastólica media y el IR durante el neumoperitoneo. (Tabla 2) En cambio, la velocidad sistólica media no experimentó un descenso significativo durante el neumoperitoneo. (Figs. 6 y 7).

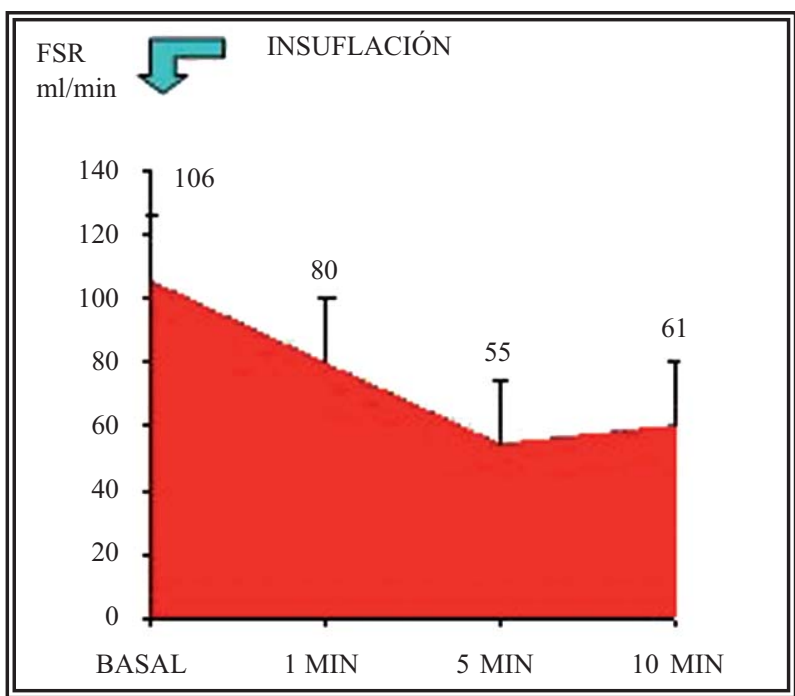

FIGURA 4. Efecto de la insuflación en el FSR durante la laparoscopia. El valor basal corresponde a la primera toma con sonda electromagnética de FSR tras la disección de la arteria renal, con neumoperitoneo establecido de $15 \mathrm{mmHg}$. Las siguientes medidas se realizan a los minutos 1, 5 y 10.

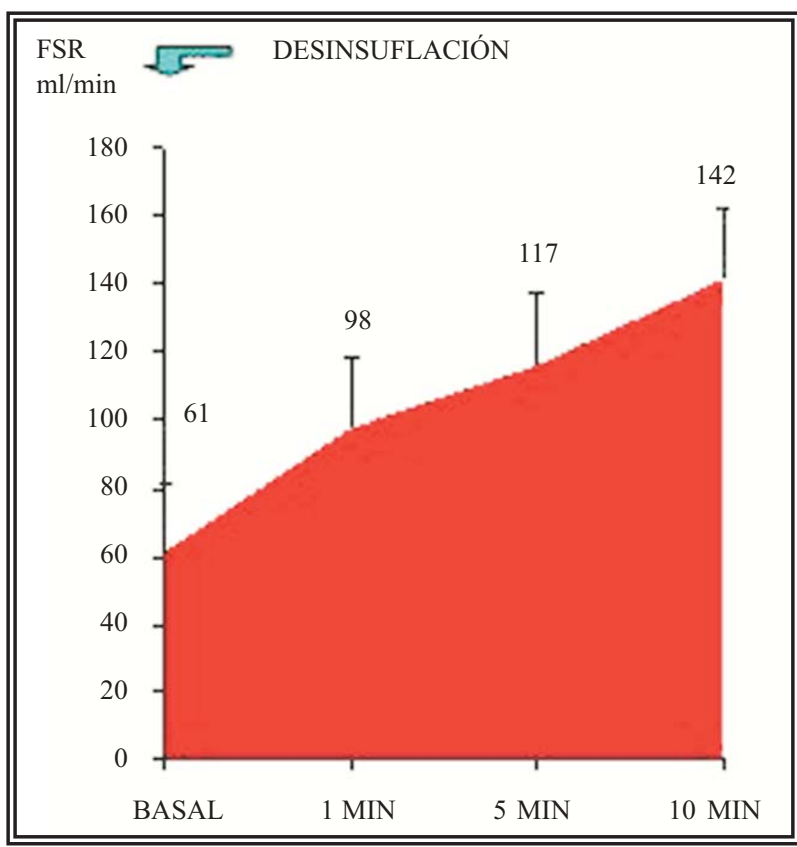

FIGURA 5. Efecto de la desinsuflación en el FSR durante la laparoscopia. El valor basal corresponde a la primera medida con sonda electromagnética de FSR, previo a iniciar la desinsuflación con neumoperitoneo de $15 \mathrm{mmHg}$. Las siguientes tomas se realizan a los minutos 1,5 y 10 de inicio de la desinsuflación.

\section{Efectos sobre el filtrado glomerular}

Por último el análisis comparativo de las modificaciones del FG y diuresis entre ambos grupos mostraba que ni la diuresis ni el FG se 
Tabla 2

Valores de perfusión renal medidos por eco-doppler, basal y a los 5 minutos de la insuflación durante la nefrectomía laparoscópica

\begin{tabular}{lccc}
\hline $\begin{array}{l}\text { Perfusión renal } \\
\text { Eco-doppler }\end{array}$ & $\begin{array}{c}\text { Basal } \\
\text { media } \pm \text { d.s. }\end{array}$ & $\begin{array}{c}\text { Neumoperitoneo } \\
\text { media } \pm \text { d.s. }\end{array}$ & $\begin{array}{c}\text { Valor } \\
\text { de p }\end{array}$ \\
\hline $\begin{array}{l}\text { Velocidad } \\
\text { sistólica }(\mathrm{cm} / \mathrm{s})\end{array}$ & $26,7 \pm 0,2$ & $20,3 \pm 0,2$ & $<0,1$ \\
$\begin{array}{l}\text { Velocidad } \\
\text { diastólica }(\mathrm{cm} / \mathrm{s})\end{array}$ & $10,1 \pm 0,2$ & $4,8 \pm 0,1$ & $<0,001$ \\
IR & $0,6 \pm 0,0$ & $0,8 \pm 0,0$ & $<0,001$ \\
\hline
\end{tabular}

modificaban de forma significativa durante la nefrectomía abierta. Sin embargo, esta reducción es significativamente mayor durante la nefrectomía laparoscópica con disminuciones porcentuales de la diuresis a los 30 y 60 minutos del 57,6\% y $58,4 \%$ respectivamente frente a las reducciones del $13,8 \%$ y $19,5 \%$ inducidas por la nefrectomía abierta en los mismos períodos de tiempo (Fig. 8). De la misma forma, resultados superponibles se encuentran respecto al FG. El FG descendió un $61,2 \%$ y $62,7 \%$ a los 30 y 60 minutos de insuflación respectivamente (Fig. 9).

\section{DISCUSIÓN}

Los valores de TAM en el grupo LAP fueron significativamente superiores que los de TAM en el grupo ABIERTO.

El aumento transitorio de la TA es probablemente causado por un incremento de la resistencia vascular periférica como consecuencia de la compresión que induce el neumoperitoneo sobre

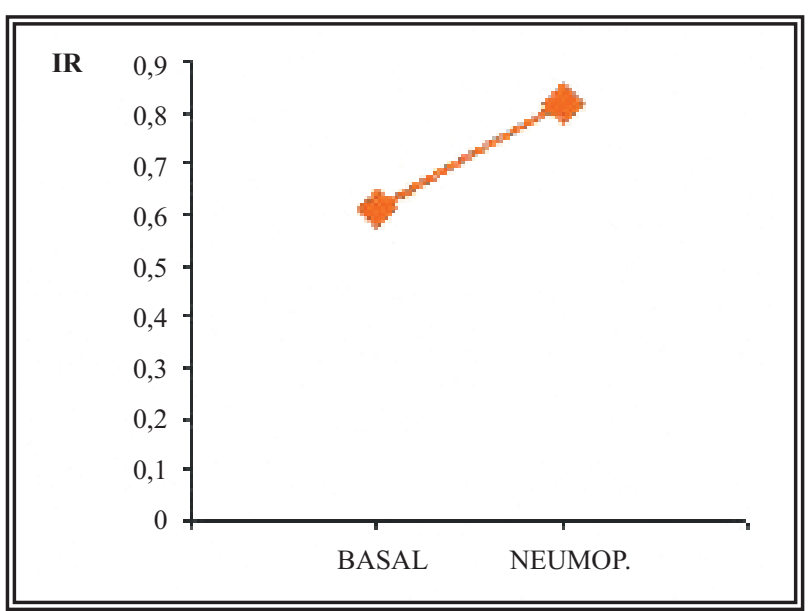

FIGURA 7. IR basal y a los 5 minutos tras el inicio de la misma.

la circulación esplácnica $6,25,27$. A medida que la insuflación de gas aumenta la PIA, la resistencia vascular sistémica se eleva. La magnitud del efecto de la PIA en la resistencia vascular sistémica como demostraron Kashtan et al. ${ }^{9}$, depende de la volemia del sujeto. La TAM es producto del gasto cardíaco y de la resistencia arterial: el equilibrio entre ambos, a una PIA $<20 \mathrm{mmHg}$, eleva la $\mathrm{TAM}^{28}$.

Pero como han señalado numerosos autores ${ }^{5,29-33}$, también jugaría un papel importante la liberación de sustancias vasoactivas, dado que el incremento de la TA no es inmediato tras la instauración del neumoperitoneo, ni vuelve rápidamente a sus valores normales tras cesar la insuflación. Se ha documentado, un aumento de dos a cuatro veces en la actividad de renina plasmática periférica, durante la insuflación intrabdominal en los modelos experimentales con ratas y cer$\operatorname{dos}^{5,30,31}$. Al descomprimir el abdomen se advirtió que los niveles de renina volvían a sus niveles basales ${ }^{29}$. Kishimoto et al. ${ }^{32}$, descubrieron un aumento al cuádruple en el índice de secreción de renina solamente a $15 \mathrm{mmHg}$. Bloomfield et al. ${ }^{31}$, demostraron, que la descompresión abdominal, reducía de forma significativa tanto la actividad de renina, como los niveles de aldosterona.
FIGURA 6. Velocidad de perfusión basal y de resistencia vascular medidos mediante eco-doppler basal, previo a la insuflación y a los 5 minutos tras el inicio de la misma. 


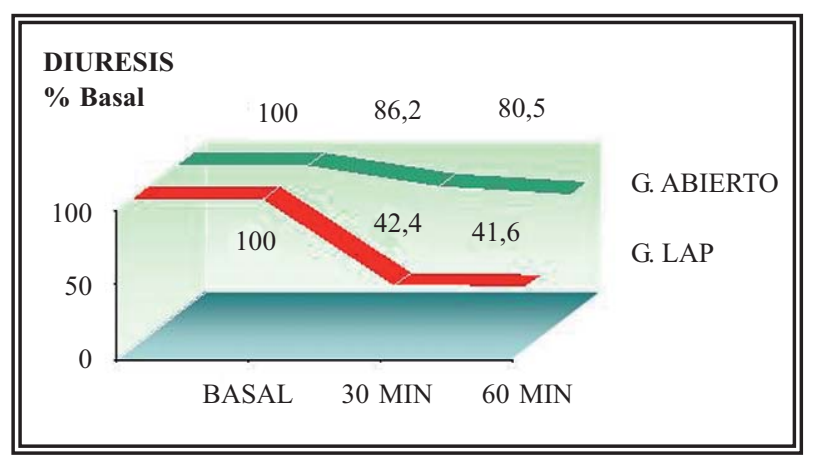

FIGURA 8. Análisis comparativo de la evolución de la diuresis durante la nefrectomía abierta y laparoscópica. Porcentaje de disminución de la diuresis respecto a la diuresis basal a los 30 y 60 minutos de cirugia.

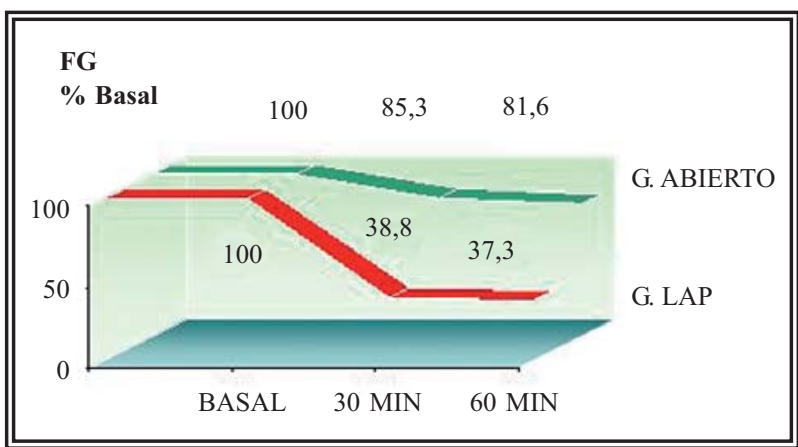

FIGURA 9. Análisis comparativo de la evolución del FG durante la nefrectomia abierta y laparoscópica. Porcentaje de disminución del FG respecto al FG basal a los 30 y 60 minutos de cirugía.

Chiu et al. ${ }^{6,23}$, en sus estudios sobre modelo experimental en cerdo, también evidenciaron un incremento significativo de la TA, y del gasto cardíaco. Koivusalo et al. ${ }^{34}$, en sus estudios realizados en pacientes sometidos a colecistectomía laparoscópica, también evidenciaron un incremento progresivo de la TAM tras 5 min de insuflación. Igualmente describieron la elevación de los niveles de adrenalina y renina, comenzando ésta última a elevarse a los 15 min de la inducción del neumoperitoneo manteniéndose estable durante toda la cirugía. Existía una correlación significativa entre los niveles de renina y los valores de TAM.

La PVC media en el grupo LAP es significativamente superior a la PVC media del grupo ABIERTO.

El incremento de la PIA durante la laparoscopia, eleva el diafragma incrementándose la presión intratorácica, que puede llegar a alcanzar el doble de la presión en la vena cava, y disminuyendo el retorno venoso. Con presiones de 15 $\mathrm{mmHg}$, los vasos, especialmente la vena cava y las venas esplácnicas son comprimidas, aunque no llegan a colapsarse ${ }^{5,6}$. Por ello, la elevación de la PVC es más precoz que la elevación de la TAM, y se mantiene en el tiempo.

Varios estudios experimentales, como los de Kashtan et al. ${ }^{9}$, y Chiu et al. $^{9}$, demuestran el mismo comportamiento hemodinámico objetivado en nuestro estudio. Koivusalo et al. ${ }^{34}$, también reflejan en sus trabajos, elevaciones del $146 \%$ de la PVC a los 5 min de la insuflación en el curso de colecistectomías laparoscópicas, manteniéndose elevada durante toda la cirugía para ir disminuyendo de una forma lentamente progresiva al término de la misma, alcanzándose el valor basal 1 hora después de su finalización.

El grupo LAP experimentó una disminución significativa del FSR respecto al FSR del grupo ABIERTO.

El FSR, es estable a una PIA por debajo de 10 mmHg. Han sido numerosos los estudios que han demostrado una reducción significativa del FSR asociado al incremento de la PIA ${ }^{6,22,23,35}$. Se ha comprobado en modelos experimentales con animal grande ${ }^{18,29}$ y pequeño ${ }^{24}$ el descenso de FSR que tiene lugar durante el neumoperitoneo. Este descenso ocurre en animales pese a estar adecuadamente expandidos, con PVC mantenidas superiores a $10 \mathrm{cmH}_{2} \mathrm{O}$ y con un gasto cardíaco estable $^{35}$. La presión que afecta al riñón, los vasos y la disminución del gasto cardíaco jugarían un papel importante.

Resultados similares a este trabajo se reflejan por otros autores como Cisek et al. ${ }^{35}$, y Chiu et al. ${ }^{6}$, que emplean un modelo animal similar. El neumoperitoneo producía una disminución del FSR del 60\% tras alcanzar una PIA de $15 \mathrm{mmHg}$, registrándose a los 30 minutos una elevación transitoria seguida de un pequeño descenso del FSR que se mantenía durante las 2 horas de duración del proceso. Pero esta alteración en la perfusión renal era reversible tras la desinsuflación, y volvía a los niveles basales inmediatamente después de la desaparición del neumoperitoneo.

El análisis secuencial del FSR a intervalos cortos de tiempo durante los procesos de insuflación 
y desinsuflación llevado a cabo en nuestro estudio, permite establecer varios hechos con potencial trascendencia clínica:

- La reducción de FSR inducida por el neumoperitoneo tiene lugar inmediatamente después de la insuflación siendo al minuto del $25 \%$ y a los 5 minutos casi del 50\%, quedándose estabilizado desde ese momento. El carácter temprano de esta reducción del FSR sugiere que el desencadenante, al menos inicial, es puramente mecánico por aumento de la PIA, sin que probablemente haya factores humorales o neuroendocrinos.

- La recuperación del FSR al cesar la insuflación tiene lugar de modo inmediato, alcanzándose al minuto prácticamente el FSR inicial (pre-insuflación), teniendo lugar en los minutos siguientes un aumento del FSR a niveles superiores a los basales. Probablemente este hecho se justifica por una vasodilatación renal en la que podrian estar involucradas sustancias vasoactivas.

- Al menos con períodos de insuflación cortos, el efecto del FSR es inmediatamente reversible, y presumiblemente, no tiene repercusión funcional trascendente en los procesos laparoscópicos convencionales. Podría plantearse, a la vista del incremento del FSR reactivo postdesinsuflación, la conveniencia durante procedimientos laparoscópicos prolongados, de llevar a cabo ciclos intermitentes de insuflación-desinsuflación con la intención de minimizar la isquemia renal relativa inducida por el neumoperitoneo, especialmente en pacientes con compromiso de la función renal.

$\mathrm{El}$ análisis de las modificaciones de la perfusión renal basal e inducida por el neumoperitoneo, evaluada por eco-doppler en el grupo LAP, reflejaron una disminución significativa de la velocidad diastólica y un incremento significativo del IR respecto a los valores basales.

La velocidad de perfusión depende del IR y de la TA. A su vez, el IR es el resultado del cociente entre: velocidad sistólica-velocidad diastólica/ velocidad sistólica. Las modificaciones hemodinámicas que se observan mediante eco-doppler durante el aumento de la PIA sufrido durante el neumoperitoneo, tienen su origen en la disminución del FSR mediado por la vasoconstricción y el incremento de la resistencia de las arterias intraparenquimatosas. Este efecto tiene más repercusión sobre la velocidad diastólica, ya que se trata de un fenómeno pasivo, que sobre la velocidad sistólica, lo que conduce a un incremento significativo del cociente y por tanto del $\mathrm{IR}^{36,37}$.

Existe por tanto, en el grupo LAP, una disminución del FSR significativa, desde los primeros 5 min de neumoperitoneo medida con sonda electromagnética, que también es objetivable con eco-doppler. Ambas medidas guardan una buena correlación temporal, ya que se evidencian a los 5 min de insuflación.

Son muchos los estudios que demuestran una buena correlación entre las modificaciones del flujo arterial y la perfusión medida por eco-doppler ${ }^{38-40}$. Chiu et al. ${ }^{6}$, empleando un medidor de flujo de doppler-láser insertado en la cortical renal, demostraron un descenso significativo de la perfusión cortical renal que se asociaba a oliguria durante las dos horas siguientes a la creación de neumoperitoneo a $15 \mathrm{mmHg}$. Otros autores como Stern et al. ${ }^{41}$, emplearon la misma técnica, microesferas radioactivas ${ }^{42}$ y eco doppler arterial renal para la medir la perfusión renal, encontrando una excelente correlación entre la perfusión renal parenquimatosa y flujo arterial renal entre los tres métodos.

No obstante, también hay algunos estudios experimentales donde la correlación entre el flujo vascular y el IR no es tan estrecha. Los trabajos de Taylor et al. ${ }^{43}$, y Siebert et al. ${ }^{44}$, en modelos experimentales caninos, muestran que la correlación entre el IR y el flujo arterial cerebral no era significativa ( $p>0,2)$, si bien, existe una relación inversamente proporcional entre la presión de perfusión y el IR en el sistema nervioso central canino. ${ }^{44}$ En cambio, la correlación con la velocidad de perfusión si alcanzaba significación estadística $(p=0,0001)^{43}$.

En relación con las modificaciones descritas del FSR, observamos que durante la disección renal en el curso de la nefrectomía, se produce una reducción de la diuresis tanto por vía abierta como laparoscópica. Sin embargo, esta reducción es significativamente mayor durante la nefrectomía laparoscópica. De la misma forma, resultados superponibles se encuentran respecto al FG. 
El incremento de la PIA se ha asociado a oliguria en numerosos estudios ${ }^{18,24,45}$. Existen publicaciones que describen casos de oliguria e incluso anuria por el mismo mecanismo en presencia de hemoperitoneo o ascitis ${ }^{18,19}$.

Page $^{15}$, fue el primero en demostrar que la compresión del parénquima renal, se relacionaba con la aparición de hipertensión renal, y con una reducción de la diuresis.

La compresión renal directa por presión intrabdominal > $10 \mathrm{mmHg}$, causa una disminución del FG y del FSR ${ }^{18}$ con la consiguiente oliguria $^{46}$.

Razvi et al. ${ }^{17}$, demostraron, que la compresión directa del parénquima renal, causaba un efecto directo y reversible, al igual que la compresión arterial y venosa renal. La compresión renal ejercida por el neumoperitoneo, afectaría al FG y también ocasionaría la compresión de las nefronas. Ambos mecanismos contribuirían a la disminución de la diuresis. Estas alteraciones funcionales no se han acompañado de modificaciones histopatológicas en las referencias existentes en la literatura ${ }^{2,28}$. Razvi et al. ${ }^{17}$, demostraron que una compresión renal a $15 \mathrm{mmHg}$, condiciona una disminución de la diuresis del 63\%, del FG en un 21\%, y del FSR en un 26\%.

En modelos experimentales en cerdo, a presiones de $20 \mathrm{mmHg}$ hay casi un 15\% de disminución del flujo arterial renal, de un 58 a un 60\% de caída de la perfusión cortical, un 64\% de reducción del FG y un 50\% de disminución de la diuresis ${ }^{6,22,23}$.

Sin embargo, cuando la compresión renal se lleva a cabo unilateralmente, la diuresis disminuye sólo un 25\%. Después de la desinsuflación, la perfusión cortical renal vuelve a sus niveles normales, aunque la diuresis continúa disminuida una hora más ${ }^{47}$. Así, la compresión renal no es el único factor causante de la oliguria, sino que actúa conjuntamente con los mecanismos mencionados.

La compresión del parénquima por sí sola, puede ser responsable de la disminución reversible del FG, del FSR y de la liberación de renina durante una insuflación laparoscópica. Pero existen otros factores implicados: la oliguria inducida por el neumoperitoneo, está igualmente influenciada por la disminución del flujo vascular renal causada por el incremento de la PVC. Kirsch et al. ${ }^{24}$, tras los estudios realizados en un modelo experimental en ratas, demostraron que con una insuflación de $10 \mathrm{mmHg}$, el flujo de la cava inferior se reducía en un $93 \%$ y el flujo aórtico en un 45\%. También la disminución del GC interviene en el proceso: Vukasin et al. ${ }^{20}$, utilizaron el modelo de perro para demostrar una disminución concomitante de la diuresis y el GC durante la insuflación con $\mathrm{CO}_{2}$ a intervalos de tiempo variables.

Si el único factor implicado en la reducción del FG fuese el mecánico como consecuencia del aumento de la PIA, sería esperable que la recuperación del FG fuese paralela a la del FSR, la cual es inmediata ${ }^{17,28,35}$. Sin embargo no es así, y la recuperación del FG tras desaparecer el neumoperitoneo se produce lentamente y parece estar en relación con factores humorales $26,35,48$. Está claramente establecido, que la función secretora del sistema yuxtaglomerular está mediada por los cambios de la presión intraluminal en la arteriola eferente ${ }^{6}$. El papel de los factores hormonales sobre el FG durante la insuflación ha sido referido por varios autores $^{5,6,9,16,18,20,22-24,27,31,32,47,49}$.

Este efecto podría venir mediado por la actividad de mediadores neuroendocrinos como la renina plasmática. Skinner ${ }^{16}$ demostró una elevación de la actividad de la renina plasmática, después de una compresión del riñón por balón a tensiones de 15 a $40 \mathrm{mmHg}$. Este incremento de la actividad de renina plasmática con el aumento de la PIA ha sido ampliamente documentado en la literatura ${ }^{17}$.

Cisek et al. ${ }^{50}$, en su modelo experimental porcino, demuestran que el incremento de la PIA se asocia con una disminución del FG, incrementándose la presión hidrostática a ambos lados de la membrana glomerular. Pero la diferencia de presiones en los vasos aferentes y eferentes no sería el único mecanismo implicado, ya que la normalización de los valores de FG, es posterior a la desaparición del neumoperitoneo. Además, el descenso de la diuresis es superior al descenso del FG, lo que sugiere que la reabsorción distal del glomérulo se incrementa con la elevación de la PIA. De nuevo, el estudio hormonal y la liberación local de mediadores cobran importancia en 
la explicación de este fenómeno. Señalan en el mismo estudio, que los valores de FG tras 1 semana de insuflación laparoscópica, no difería de manera significativa con el valor de FG previo a la misma.

También Chiu et al. ${ }^{51}$, demuestran una lenta normalización de la diuresis 1 hora después de cesar el neumoperitoneo, lo que de nuevo hace pensar que la compresión parenquimatosa no es el único factor que induce la alteración en la función renal. Otros factores han sido descritos en la literatura desde hace ya más de dos décadas, a pesar de lo cual su papel no está claramente establecido. Entre ellos se encuentran las elevaciones de ADH. Endotelina (ET) y Aldosterona ${ }^{24,31,33}$ a través fundamentalmente del Sistema Renina-Angiotensina-Aldosterona. Asimismo la actividad adrenérgica mediada por las catecolaminas, y las modificaciones del FSR, jugarían un papel fundamental. Stowe et al. ${ }^{52}$, observaron en un modelo de neumoperitoneo en rata, que el uso de antagonistas de los receptores de la ET, atenuaba la caída del FG, siendo esta reducción del $53 \%$ en los animales tratados frente al 88\% para los animales del grupo control que no recibieron antagonistas de los receptores de ET.

El conjunto de modificaciones que el neumoperitoneo induce sobre el FSR, el FG y la diuresis pueden tener repercusión sobre el sindrome de isquemia-reperfusión que acontece al revascularizarse el injerto tras el TR, así como en la función inmediata del mismo ${ }^{53}$.

\section{CONCLUSIONES}

Durante la nefrectomía laparoscópica, se produce un incremento significativo de la TAM y de la PVC respecto a la TAM y PVC durante la nefrectomía abierta.

El FSR durante la nefrectomía laparoscópica experimenta un descenso significativo respecto a la nefrectomía abierta. El eco-doppler demuestra una disminución significativa de la velocidad diastólica y un incremento significativo del índice de resistencia respecto a situación basal durante el neumoperitoneo, que se correlaciona con la medida del FSR. Ello condiciona una disminución significativa de la diuresis que es paralela a la disminución del FG.

\section{REFERENCIAS}

11. Lee CM. Acute hypotension during laparoscopy: A case report. Anesth Analg. 1975; 54(1):142-143.

12. Hazebroek E, De Bruin R, Bouvy N, Marquet RL, Bonthuis F, Bajema IM, et al. Long-term impact of neumoperitoneum used for laparoscopic donor nephrectomy on renal function and histomorphology in donor and recipient rats. Ann Surg. 2003; 237(3):351-357.

13. Hashikura Y, Kawasaki S, Munakata Y, Hashimoto S, Hayashi K, Makuuchi M. Effects of peritoneal insufflation on hepatic and renal blood flow. Surg Endosc. 1994; 8(7):759-761.

14. Eleftheriadis E, Kotzampassi K, Botsios D, Tzartinoglou E, Farmakis H, Dadoukis J. Splachnic ischemia during laparoscopic cholecystectomy. Surg Endosc. 1996;10(3):324-326.

15. Koivusalo AM, Kellokumpu I, Scheinin M, Tikkanen I, Halme L, Lindgren L. Randomized comparison of the neuroendocrine response to laparoscopic cholecystectomy using either conventional or abdominal wall lift techniques. Br J Surg. 1996; 83(11):1532-1536..

16. Chiu AW, Chang LS, Birkett DH, Babayan RK. The impact of pneumoperitoneum, pneumoretroperitoneum, and gasless laparoscopy on the systemic and renal hemodynamics. J Am Coll Surg. 1995;181(5):397-406.

17. Ho HS, Saunders CJ, Gunther RA, Wolfe BM. Effector of hemodynamics during laparoscopy: $\mathrm{CO} 2$ absorption or intraabdominal pressure?. J Surg Res. 1995;59(4):497-503.

18. Safran DB, Orlando R $3^{\text {rd }}$. Physiologic effects of pneumoperitoneum. Am J Surg. 1994; 167(2):281-286.

19. Kashtan J, Green, JF, Parsons EQ. Hemodynamic effects of increased abdominal pressure. J Surg Res. 1981;30(3):249255.

10. Hunter JG. Laparoscopic pneumoperitoneum: the abdominal compartment syndrome revisited. J Am Coll Surg. 1995;181 (5):469-470.

11. Lindgren L, Koivusalo A-M, Kellokumpu I. Convencional pneumoperitoneum compared with abdominal wall lift for laparoscopic cholecystectomy. Br J Anaesth. 1995; 75(5):567-572.

12. Mäkinen MT, Yli-Hankala A. The effect of laparoscopic cholecystectomy on respiratory compliance as determined by continuous spirometry. J Clin Anaesth. 1996; 8(2):119-122.

13. Guler C, Sade M, Kirkali $Z$. Renal effects of carbon dioxide insufflation in rabbit pneumoretroperitoneum model. J Endourol. 1998;12(4):367-370.

14. Kheirabadi BS, Tuthill D, Pearson R, Bayer V, Beall D, Drohan $\mathrm{W}$ et al. Metabolic and hemodynamic effects of $\mathrm{CO} 2$ pneumoperitoneum in a controlled hemorrhage model. J Trauma. 2001;50(6):1031-1043.

15. Engel WJ, Page I. The production of persistent arterial hypertension by cellophane perinephritis. JAMA. 1939;113:2046-2048.

16. Skinner SL, McCubbin JW, Page IH. Control of renin secretion. Cir Res. 1964;15: 54-76.

17. Razvi HA, Fields D, Vargas JC, Vaughan ED Jr, Vukasin A, Sosa RE. Oliguria during laparoscopic surgery: evidence for direct renal parenchymal compression as an etiologic factor. J Endourol. 1996;10(1): 1-4.

18. Harman PK, Kron IL, McLachlan HD, Freedlender AE, Nolan SP. Elevated intraabdominal pressure and renal function. Am Surg. 1982;196(5):594-597.

19. Eggleton MG, Pappenherimer JR, Winton FR. The influence of diuretics on the osmotic work done and on the efficiency of the isolated kidney of the dog. J Physiol.1940;97(3):363-382.

20. Johannsen G, Andersen M, Juhl B, et al. The effect of general anaesthesia on the haemodynamic events during laparoscopy with CO2 insuflation. Acta Anaes Scnd 1989;33:132-136. 
21. McDougall EM, Monk TG, Wolf JS Jr, Hicks M, Clayman RV, Gardner S, et al. The effect of prolonged pneumoperitoneum on renal function in animal model. J Am Coll Surg. 1996;182(4):317-328.

22. Cisek LJ, Gobet RM, Peters CA. Pneumoperitoneum produces reversible renal dysfunction in animals with normal and chronically reduced renal function. J Endourol 1998; 12(2):95-100.

23. Chiu AW, Azadzoi KM, Hatzichristou DG, Siroky MB, Krane RJ, Babayan RK. Effects of intra-abdominal pressure on renal tissue perfusion during laparoscopy. J Endourol. 1994;8(2): 99-103.

24. Kirsch AJ, Hensle TW, Chang DT, Kayton ML, Olsson CA, Sawczuk IS. Renal effects of $\mathrm{CO} 2$ insufflation: Oliguria and acute renal dysfunction in a rat pneumoperitoneum model. Urology. 1994,43(4): 453-459.

25. Iwase K, Takenaka H, Yagura A, Ishizaka T, Ohata T, Takagaki $\mathrm{M}$, et al. Hemodynamic changes during laparoscopic cholecystectomy in patients with heart disease. Endoscopy. 1992; 24(9):771-773.

26. Flowers JL, Jacobs S, Cho E, Morton A, Rosenberger WF, Evans D, et al. Comparison of open and laparoscopic live donor nephrectomy. Ann Surg. 1997;226(4):483-489.

27. Ekman LG, Abrahamsson J, Biber B, Forssman L, Milsom I, Sjoqvist BA. Hemodynamic changes during laparoscopy with positive end-expiratory pressure ventilation. Acta Anaes Scand. 1988;32(6): 447-453.

28. Lindström P, Wadström J, Ollerstam A, Johnsson C, Persson AE. Effects of increased intra-abdominal pressure and volume expansion on renal function in the rat. Nephrol Dial Transplant 2003; 18(11): 2269-2277.

29. London E, Ho H, Neuhaus Ann, Wolfe BM, Rudich SM, Perez RV. Effect of intravascular volume expansion on renal function during prolonged CO2 pneumoperitoneum. Ann Surg 2000; 231(2):195-201.

30. O’Leary E, Hubbard K, Tormey W, Cunningham AJ: Laparoscopic cholecystectomy after pneumoperitoneum and changes in position. Br J Anaesthesia. 1996; 76(5):640-644.

31. Bloomfield GI, Blocher CR, Fakhry IF, Sica DA, Sugerman HJ. Elevated intrabdominal pressure increases plasma renin activity and aldosterona levels. J Trauma 1997; 42(6):997-1004.

32. Kishimoto T, Maedawa M, Age Y, Yamamoto, K. Intrarenal distribution of blood flow and renin release during renal venous pressure elevation. Kidney Inter 1973;4(4):259-266.

33. Diebel LN, Wilson RF, Dulchavsky SA, Saxe J. Effects of increased intrabdominal pressure on hepatic arterial, portal venous, and hepatic microcirculatory blood flow. J Trauma. 1992;33(2):279-283.

34. Koivusalo AM, Kellokumpu I, Scheinin M, Tikkanen I, Makisalo H, Lindgren L. A Comparison of gasless and conventional carbon dioxide pneumoperitoneum methods for laparoscopic cholecystectomy. Anesth Analg. 1998; 86(1):153158.

35. Cisek L, Gobet R, Peters C. Pneumoperitoneum produces reversible renal dysfunction in animals with normal and chronically reduced renal function. J Endourol. 1998; 12(2):95100.

36. Cole T, Brock J, Pope J, Schrum FF, Milam DF, Flickinger JE, et al. Evaluation of renal resistive index, maximum velocity, and mean arterial flow velocity in a hydronephrotic partially obstructed pig model. Invest Rad. 1997;32(3):154-160.

37. Mastorakou I, Robbins MEC, Bywaters T. Resistance and pulsatility doppler indices: how accurately do they reflect changes in renal vascular resistance. Br J Radiol.1993; 66(787): $557-580$.
38. Sahn DJ. Instrumentation and Physical factors related to visualization of stenotic and regurgitant jets by doppler and color flow mapping. J Am Coll Cardiol. 1998; 12(5):1354-1365.

39. Jain AP, Fan PH, Philpot EF, Nanda NC, Aggarwal KK, Moos $\mathrm{S}$, et al. Influence of various instrument settings on the flow information derived from the power mode. Ultrasound Med Biol 1991;17(1):49-54.

40. Durick JE, Winter TC 3rd, Schmiedl UP, Cyr DR, Starr FL 3rd, Mack LA. Renal perfusion: Pharmacological changes depicted with power doppler US in an animal model. Radiology. 1995; 197(3):615-617.

41. Stern MD, Bowen PD, Parma R, Osgood RW, Bowman RL, Stein JH. Measurement of renal cortical and medullary blood flow by laser-Doppler spectroscopy in the rat. Am J Physiol. 1979; 236(1):80-87.

42. Caldwell CB, Ricotta JJ. Changes in visceral blood flow with elevated intraabdominal pressure. J Surg Res. 1987; 43(1):1420.

43. Taylor GA, Short BL, Walker LK, Traystman RJ. Intracranial blood flow: Quantification with duplex Doppler and color Doppler flow US. Radiology. 1990; 176(1): 231-236.

44. Seibert JJ, McCowan TC, Chadduck WM, Adametz JR, Glasier CM, Williamson SL, et al. Duplex pulsed Doppler US versus intracranial pressure in the neonate: Clinical and experimental studies. Radiology. 1989;171(1):155-159.

45. Kerbl K, Clayman RV, McDougall EM, Kavoussi LR. Laparoscopic nephrectomy: the Washington University experience. Br J Urol.1994; 73(3):231-236.

46. Kalk H. Erfahrung mit der Laparoskopie (zugleich mit Beschreibung eines neuen Instrumentes). Z Clin Med. 1929; 111:303-348.

47. Hamilton BD, Chow GK, Stowe NT. Increased intra-abdominal pressure during pneumoperitoneaum stimulates endothelin release in a canine model. J Endourol 1998;12(2):193-197.

48. Wilhelm SM, Simonson MS, Robinson AV, Stowe NT, Schulak JA. Endothelin up-regulation and localization following renal ischemia and reperfusion. Kidney Int. 1999; 55(3):1011-1018.

49. Brundell S.M, Tsopelas C, Chatterton B, Touloumtzoglou J, Hewett PJ. Experimental study of peritoneal blood flow and insufflation pressure during laparoscopy. Br J Surg. 2002; 89(5):617-622.

50. Brown JM, Grosso MA, Terada LS, Whitman GJ, Banerjee A, White CW, et al. Endotoxin pretreatment increases endogenous myocardial catalase activity and decreases ischemiareperfusion injury of isolated rat hearts. Proc Natl Acad Sci. USA 1989; 86(7):2516-2520.

51. Chiu AW, Chang LS, Birkett DH, Babayan RK. Changes in urinary output and electrolytes during gaseous and gasless laparoscopy. Urol Res.1996; 24(6):361-366.

52. Ambrose JA, Onders RP, Stowe NT, et al. Pneumoperitoneum upregulates preproendothelin-1 messenger RNA. Surg Endosc. 2001;15(2):183-188.

53. Burgos FJ, Pascual J, Briones B, Cuevas B, Villafruela J, Correa $\mathrm{C}$, et al. Influence of laparoscopic live donor nephrectomy in ischemia-reperfusion syndrome and renal function after kidney transplantation: an experimental study. Transplant Proc. 2003;35(5):1664-1665.

Correspondencia autora: Dra. A.I. Linares Quevedo Servicio de Urología. Hospital de Fuenlabrada. Camino del Molino, s/n. 28942 Fuenlabrada. Madrid E-mail autora: analinaresquevedo@hotmail.com Información artículo: Original - Trabajo experimental Trabajo recibido: junio 2006

Trabajo aceptado: enero 2007 